\title{
Some New Characterizations of Symplectic Curve in 4-Dimensional Symplectic Space
}

\author{
Esra Çiçek Çetin ${ }^{1}$, Mehmet Bektaş²
}

\begin{abstract} this characterizations for Minkowski space and obtained very different results. 4-Dimensional Symplectic Space and we give some characterizations and theorems for these curves.

Keywords: Symplectic curve, Symplectic space

2010 AMS: 53A04, 53B25

${ }^{1}$ Department of Mathematics, Firat University, 23119 Elazı̆g, Türkiye, ORCID: 0000000182130156

${ }^{2}$ Department of Mathematics, Firat University, 23119 Elazığ, Türkiye , ORCID: 0000000257974944

*Corresponding author:mbektas@firat.edu.tr.

Received: 05 November 2019, Accepted: 12 December 2019, Available online: 329 December 2019
\end{abstract}

It is well known that there exist characterizations for curve in Euclidean space. Also, a lot of authors extended

In this paper, we introduce the geometric properties of Symplectic Curve in 4-Dimensional Symplectic Space which given by [1, 2]. Later we obtained the conditions for Symplectic Curve to lie on some subspaces of

\section{Introduction}

Symplectic geometry provided a language for clasical mechanics. Through its recent huge development, it conquered an independent and rich territory, as a central branch of differantial geometry and topology. On the other hand Symplectic geometry is mathematical apparatus of such areas of Physics as classical mechanics, geometrical optics and thermodynamics. In order words Symplectic geometry arose from the study of optics and mechanics.

The study of local symplectic invariants of curves in affine symplectic geometry was initialed by Chern and Wang [3]. The issue has however remained silent for many years, before being taken up on several occasions in recent literature $[4,5,6,7]$. Recently, Kamran et al. [1] developed the results and obtained explicitly the symplectic invariants and Frenet frames for curves in affine symplectic geometry by successively differentiating the tangent vector of the curve and using the non-degenerate inner product associated to the symplectic form. Frenet frame and Frenet formulae of curves by using the equivariant moving frame method due to Fels and Olver [8, 9].

In the present paper, firstly we give a short view of the basis of symplectic curves in the 4-dimensional symplectic space and secondly we investigate the conditions for symplectic curves to lie on some subspaces of $\mathbb{R}^{4}$ and we give some characterizations and theorems for symplectic curves.

\section{Preliminaries}

Let us give brief related to symplectic space. One can found a brief account of the symplectic space in $[1,2]$. The symplectic space $\operatorname{Sim}=\left(\mathbb{R}^{4}, \Omega\right)$ is the vector space $\mathbb{R}^{4}$ endowed with the standard symplectic form $\Omega$, given in global Darboux coordinates 
by $\Omega=\sum_{i=1}^{2} d x_{i} \wedge d y_{i}$. Each tangent space is endowed with symplectic inner product defined in canonical basis by

$$
\langle u, v\rangle=\Omega(u, v)=x_{1} \eta_{1}+x_{2} \eta_{2}-y_{1} \xi_{1}-y_{2} \xi_{2}
$$

where $u=\left(x_{1}, x_{2}, y_{1}, y_{2}\right)$ and $v=\left(\xi_{1}, \xi_{2}, \eta_{1}, \eta_{2}\right)$.

A symplectic frame is a smooth section of the bundle of linear frames over $\mathbb{R}^{4}$ which assigns to every point $z \in \mathbb{R}^{4}$ an ordered basis of tangent vectors $a_{1}, a_{2}, a_{3}, a_{4}$ with the property that

$$
\begin{aligned}
& \left\langle a_{i}, a_{j}\right\rangle=\left\langle a_{2+i}, a_{2+j}\right\rangle=0,1 \leq i, j \leq 2, \\
& \left\langle a_{i}, a_{2+j}\right\rangle=0,1 \leq i \neq j \leq 2, \\
& \left\langle a_{i}, a_{2+i}\right\rangle=1,1 \leq i \leq 2 .
\end{aligned}
$$

Let $z(t): \mathbb{R} \rightarrow \mathbb{R}^{4}$ denotes a local parametrized curve. In our notation, we allow $z$ to be defined on an open interval of $\mathbb{R}$. As it is customary in classical mechanics, we use the notation $z$ to denote differentiation with respect to the parameter t:

$$
\dot{z}=\frac{d z}{d t} \text {. }
$$

Definition 2.1. A curve $z(t)$ is said to be symplectic regular if it satisfies the following non-degeneracy condition

$$
\langle\dot{z}, \ddot{z}\rangle \neq 0, \text { for all } t \in \mathbb{R} \text {. }
$$

With no loss of generality, the left-hand side of (2.1) may be assumed positive.

Definition 2.2. Let $t_{0} \in \mathbb{R}$, then the symplectic arc length $s$ of a symplectic regular curve starting at $t_{0}$ is defined by

$$
s(t)=\int_{t_{0}}^{t}\langle\dot{z}, \ddot{z}\rangle^{1 / 3} d t \text { for } t \geq t_{0} .
$$

Taking the extrerior differential of (2.2) we obtain the symplectic arc length element as

$$
d s=\langle\dot{z}, \ddot{z}\rangle^{1 / 3} d t .
$$

Dually, the arc length derivative operator is

$$
D=\frac{d}{d s}=\langle\dot{z}, \ddot{z}\rangle^{-1 / 3} \frac{d}{d t} \text {. }
$$

In the following, primes are used to denote differentiation with respect to the symplectic arc length derivative operator (2.3)

$$
z^{\prime}=\frac{d z}{d s}
$$

Definition 2.3. A symplectic regular curve is parametrized by symplectic arc length if

$$
\langle\dot{z} ; \ddot{z}\rangle=1,
$$

for all $t \in \mathbb{R}$.

Let $z(s)$ be a symplectic regular curve in $\operatorname{Sim}=\left(\mathbb{R}^{4}, \Omega\right)$. In this case there exists only one Frenet frame $\left\{a_{1}(s), a_{2}(s), a_{3}(s), a_{4}(s)\right\}$ for which $z(s)$ is a symplectic regular curve with Frenet equations

$$
\begin{aligned}
a_{1}^{\prime}(s) & =a_{3}(s) \\
a_{2}^{\prime}(s) & =H_{2}(s) a_{4}(s) \\
a_{3}^{\prime}(s) & =k_{1}(s) a_{1}(s)+a_{2}(s) \\
a_{4}^{\prime}(s) & =a_{1}(s)+k_{2}(s) a_{2}(s)
\end{aligned}
$$

where $H_{2}(s)=$ constant $(\neq 0), k_{1}(s), k_{2}(s)$ are symplectic curvatures of $z(s)$ [1]. 


\section{The characterizations of symplectic curve in 4-dimensional symplectic space}

In this section we will investigate some characterizations of symplectic curve to lie on some subspaces of 4-Dimensional Symplectic Space. In the following, we use notations and concepts from [10], unless otherwise stated

Case 1 : We will investigate the conditions under which symplectic curve z lies on the subspace spanned by $\left\{a_{3}(s), a_{4}(s)\right\}$. In this case we can write

$$
z(s)=\lambda(s) a_{3}(s)+\mu(s) a_{4}(s)
$$

for some differentiablae functions $\lambda$ and $\mu$ of the parameter s. Diffrentiating (3.1) with respect to $\mathrm{s}$

$$
z^{\prime}(s)=\lambda^{\prime}(s) a_{3}(s)+\mu^{\prime}(s) a_{4}(s)+\lambda(s) a_{3}^{\prime}(s)+\mu(s) a_{4}^{\prime}(s)
$$

and by using Frenet equations we find that

$$
z^{\prime}(s)=\left(\lambda(s) k_{1}(s)+\mu(s)\right) a_{1}(s)+\left(\lambda(s)+\mu(s) k_{2}(s)\right) a_{2}(s)+\lambda^{\prime}(s) a_{3}(s)+\mu^{\prime}(s) a_{4}(s)
$$

Since $\left\{a_{1}(s), a_{2}(s), a_{3}(s), a_{4}(s)\right\}$ is a Frenet frame we have the following equations.

$$
\begin{aligned}
& \lambda^{\prime}(s)=0 \\
& \mu^{\prime}(s)=0 \\
& \lambda(s) k_{1}(s)+\mu(s)=1 \\
& \lambda(s)+\mu(s) k_{2}(s)=0
\end{aligned}
$$

From $\lambda^{\prime}(s)=0$ and $\mu^{\prime}(s)=0$ we find that $\lambda(s)=c_{1}$ and $\mu(s)=c_{2}$. Thus we have the following theorem.

Theorem 3.1. A symplectic curve $z$ in $R^{4}$ lies on the subspace spanned by $\left\{a_{3}(s), a_{4}(s)\right\}$ if and only if it is in the form

$$
z(s)=c_{1} a_{3}(s)+c_{2} a_{4}(s)
$$

where $k_{1}=$ const.,$k_{2}=$ const. and $k_{1}(s)=\frac{1}{k_{2}(s)}, c_{1}, c_{2}$ are constants.

Case 2: We will investigate the conditions under which symplectic curve z lies on the subspace spanned by $\left\{a_{1}(s), a_{3}(s), a_{4}(s)\right\}$. In this case we can write

$$
z(s)=\lambda(s) a_{1}(s)+\mu(s) a_{3}(s)+\gamma(s) a_{4}(s)
$$

for some differentiable functions $\lambda, \mu$ and $\gamma$ of the parameter s. Differentiating (3.2) with respect to $\mathrm{s}$

$$
z^{\prime}(s)=\lambda^{\prime}(s) a_{1}(s)+\mu^{\prime}(s) a_{3}(s)+\gamma^{\prime}(s) a_{4}(s)+\lambda(s) a_{1}^{\prime}(s)+\mu(s) a_{3}^{\prime}(s)+\gamma(s) a_{4}^{\prime}(s)
$$

and by using Frenet equations we find that

$$
\begin{aligned}
z^{\prime}(s)= & \left(\lambda^{\prime}(s)+\mu(s) k_{1}(s)+\gamma(s)\right) a_{1}(s)+\left(\mu(s)+\gamma(s) k_{2}(s)\right) a_{2}(s) \\
& +\left(\mu^{\prime}(s)+\lambda(s)\right) a_{3}(s)+\gamma^{\prime}(s) a_{4}(s)
\end{aligned}
$$

Since $\left\{a_{1}(s), a_{2}(s), a_{3}(s), a_{4}(s)\right\}$ is a Frenet frame we have the following equations.

$$
\begin{aligned}
& \lambda^{\prime}(s)+\mu(s) k_{1}(s)+\gamma(s)=1 \\
& \mu(s)+\gamma(s) k_{2}(s)=0 \\
& \mu^{\prime}(s)+\lambda(s)=0 \\
& \gamma^{\prime}(s)=0
\end{aligned}
$$

We obtain that $\gamma(s)=c, \mu(s)=-c k_{2}(s)$ and $\lambda(s)=c k_{2}^{\prime}(s)$. Thus we have the following theorem. 
Theorem 3.2. A symplectic curve $z$ in $R^{4}$ lies on the subspace spanned by $\left\{a_{1}(s), a_{3}(s), a_{4}(s)\right\}$ if and only if it is in the form

$$
z(s)=c k_{2}^{\prime}(s) a_{1}(s)-c k_{2}(s) a_{3}(s)+c a_{4}(s)
$$

where $k_{2}(s) \neq 0, c$ is constant.

Case 3: We will investigate the conditions under which symplectic curve z lies on the subspace spanned by $\left\{a_{2}(s), a_{3}(s), a_{4}(s)\right\}$. In this case we can write

$$
z(s)=\lambda(s) a_{2}(s)+\mu(s) a_{3}(s)+\gamma(s) a_{4}(s)
$$

for some differentiable functions $\lambda, \mu$ and $\gamma$ of the parameter s. Differentiating (3.3) with respect to $\mathrm{s}$

$$
z^{\prime}(s)=\lambda^{\prime}(s) a_{2}(s)+\mu^{\prime}(s) a_{3}(s)+\gamma^{\prime}(s) a_{4}(s)+\lambda(s) a_{2}^{\prime}(s)+\mu(s) a_{3}^{\prime}(s)+\gamma(s) a_{4}^{\prime}(s)
$$

and by using Frenet equations we find that

$$
\begin{aligned}
z^{\prime}(s)= & \left(\mu(s) k_{1}(s)+\gamma(s)\right) a_{1}(s)+\left(\lambda^{\prime}(s)+\mu(s)+\gamma(s) k_{2}(s)\right) a_{2}(s) \\
& +\mu^{\prime}(s) a_{3}(s)+\left(\gamma^{\prime}(s)+\lambda(s) H_{2}(s)\right) a_{4}(s)
\end{aligned}
$$

Since $\left\{a_{1}(s), a_{2}(s), a_{3}(s), a_{4}(s)\right\}$ is a Frenet frame we have the following equations.

$$
\begin{aligned}
& \mu(s) k_{1}(s)+\gamma(s)=1 \\
& \lambda^{\prime}(s)+\mu(s)+\gamma(s) k_{2}(s)=0 \\
& \mu^{\prime}(s)=0 \\
& \gamma^{\prime}(s)+\lambda(s) H_{2}(s)=0
\end{aligned}
$$

Here $\mu(s)=c_{1}$ and by using equations $\mu(s) k_{1}(s)+\gamma(s)=1$ and $\lambda^{\prime}(s)+\mu(s)+\gamma(s) k_{2}(s)=0$, we find $\gamma(s)=1-c_{1} K_{1}(s)$ and

$\lambda(s)=\int\left[c-\left(1-c_{1} k_{1}(s)\right) k_{2}(s) d s\right]+c_{2}$

$$
\gamma(s)=\int\left[\left[-\int\left(-c-\left(1-k_{1}\right) k_{2}\right) d s+c_{2}\right] H_{2}(s)\right] d s+c_{3}
$$

Thus we have the following theorem.

Theorem 3.3. A symplectic curve $z$ in $R^{4}$ lies on the subspace spanned by $\left\{a_{2}(s), a_{3}(s), a_{4}(s)\right\}$ if and only if it is in the form

$$
z(s)=-\frac{c_{1}}{H_{2}(s)} k_{1}^{\prime}(s) a_{2}(s)+c_{1} a_{3}(s)+\left(1-c_{1} k_{1}(s)\right) a_{4}(s)
$$

or

$$
z(s)=\left[\int\left[c_{1}\left(k_{1}(s) k_{2}(s)-1\right)-k_{2}(s)\right] d s+c_{2}\right] a_{2}(s)+c_{1} a_{3}(s)+\left(1-c_{1} k_{1}(s)\right) a_{4}(s)
$$

where $c_{1}, c_{2}$ are constants.

\section{References}

[1] N. Kamran, P. Olver, K. Tenenblat, Local symplectic invariants for curves, Commun. Contemp. Math., 11(2) (2009), 165-183.

[2] F. Valiquette, Geometric affine symplectic curve flows in R4, Differ. Geom. Appl., 30(6) (2012), 631-641.

[3] S. S. Chern, H. C. Wang, Differential geometry in symplectic space. I, Sci. Rep. Nat. Tsing Hua Univ., 4 (1947), 453-477.

[4] J.C. Álvarez Paiva, C. E. Durán, Geometric invariants of fanning curves, Adv. Appl. Math., 42(3) (2009), 290-312.

[5] V. Deconchy, Hypersurfaces in symplectic affine geometry, Differ. Geom. Appl., 17(1) (2002), 1-13.

[6] E. Musso, L. Nicolodi, Symplectic applicability of Lagrangian surfaces, Sigma, 5 (2009), 067, 18 pages.

[7] E. Musso, E. G. Hubert, Lagrangian Curves in a 4-Dimensional Affine Symplectic Space, Acta Appl. Math., 134(1) (2014) 133-160.

[8] M. Fels, P. J. Olver, Moving coframes, I. A practic algorithm, Acta Appl. Math., 51 (1998), 161-213.

[9] M. Fels, P.J. Olver, Moving coframes, II. regularization and theoretical foundation, Acta Appl. Math., 55 (1999), 127-208.

[10] M. A. Akgün, A.İ. Sivridağ, E. Kılıç, The characterizations of a Spacelike Curve in $R_{1}^{4}$, Konuralp J. Math., 4(2) (2016), 299-307. 